\title{
Near-Threshold Ionization of Argon by Positron Impact
}

\author{
T. J. Babij, J. R. Machacek, D. J. Murtagh, S. J. Buckman, and J. P. Sullivan" \\ Plasma Research Laboratory, Research School of Physics and Engineering, Australian National University, \\ Canberra ACT 2601, Australia
}

(Received 4 October 2017; published 13 March 2018)

\begin{abstract}
The direct single-ionization cross section for Ar by positron impact has been measured in the region above the first ionization threshold. These measurements are compared to semiclassical calculations which give rise to a power law variation of the cross section in the threshold region. The experimental results appear to be in disagreement with extensions to the Wannier theory applied to positron impact ionization, with a smaller exponent than that calculated by most previous works. In fact, in this work, we see no difference in threshold behavior between the positron and electron cases. Possible reasons for this discrepancy are discussed.
\end{abstract}

DOI: 10.1103/PhysRevLett.120.113401

The energy dependence of the electron impact ionization cross section for the single ionization of atoms near the threshold is given by the Wannier Law [1], which uses a semiclassical approximation to describe the two-electron escape from a residual positive ion. In this case, the electrons escape due to correlated motion along the socalled "Wannier ridge," maintaining equal distances on opposite sides of the ion, thus effectively providing the mutual shielding required for double escape with low residual energy. The Wannier law for two-electron escape has been well established by many experimental (e.g., Refs. [2-4]) and theoretical studies (e.g., Refs. [1,5,6]). Here Wannier [1] determined semiclassically that for impact energies $E_{m}$ just above the ionization energy threshold $E_{i}$, the energy dependence of the cross section for double-electron escape from the residual positive ion core arises from the long-range Coulomb interactions of the particles. This was derived classically, and the dependence was found, as a function of excess energy, to be

$$
Q_{i}^{-} \propto\left(E_{m}-E_{i}\right)^{n} .
$$

For electron impact ionization, where the residual ion was singly charged, the exponent was found to be $n=1.127$. The range of validity of the Wannier approach is commonly accepted to be within $2 \mathrm{eV}$ of the ionization threshold [3].

The Wannier law for electron impact was later extended to positron impact ionization by Klar [7] and Grujic [8], where the calculated exponent was found to be 2.65 for the analogous process - $\mathrm{a}$ free electron and positron escaping from a residual singly charged ion. In the case of electrons, in the double escape process studied by Wannier, the escaping particles are ejected "back to back" and with equal energies (velocities). In Klar's treatment, the expected postcollision geometry is the ejected electron following a collinear path between the positron and the residual ion, with a velocity half that of the positron to maintain equidistance with the electron between the two positive charges. Further theoretical developments subsequently followed the work of Klar (e.g., Refs. [9-14]), including a quantum-mechanical treatment of the problem, by Temkin, that results in a relationship for the threshold to the excess energy that is not a simple power law, but rather a modulated linear threshold law $[9,10,12]$. This modulated linear law was based on the Coulomb-dipole (C-D) theory near threshold, wherein for the case of electron impact, the faster electron sees a dipole moment formed by the slower electron and the ion, whereas the slow electron will directly see the potential from the residual ion. It is claimed that this law applies to both positron and electron impacts, and that it gives rise to the same threshold behavior for double escape to the continuum for each projectile.

There has been some experimental work towards exploring this regime using positron impact, notably by Ashley et al. [15], who measured $e^{+}$-ion pairs in coincidence for energies up to $10 \mathrm{eV}$ above the threshold, and found exponents of $1.99 \pm 0.19$ and $1.70 \pm 0.11$ for $\mathrm{He}$ and $\mathrm{H}_{2}$, respectively, over the energy range from $1-3 \mathrm{eV}$ above the threshold. Other previous experiments using timeof-flight techniques suggested that positron and electron impact may result in the same exponent $[16,17]$. However, due to large energy spreads in the incident positron beams, these measurements were arguably outside the range in which we might expect the Wannier threshold behavior to apply. The lowest excess energy measured by Asheley et al. [15] is $1 \mathrm{eV}$ above the threshold, with an energy spread of $0.5 \mathrm{eV}$. Sueoka et al. [17] were only able to measure excess energies of $1.6 \mathrm{eV}$ and higher, potentially well above the range of validity, and Knudsen et al. [16] were limited by an energy spread of around $1 \mathrm{eV}$.

Whether it is appropriate to apply the same validity conditions to both electron and positron impact ionization 
has been disputed. Grujic [8] suggests that, due to polarization forces being much larger for positron impact than for electron impact, the region of validity for the threshold law for positron impact is larger than that for electron impact. Klar [18] speculated that extending the theory to include screening effects by the remaining electrons may extend the range of validity. In the second case, the calculation was for electron impact ionization, but the same argument may extend to positron impact ionization. Feagin [19] claims that the validity range will depend on the final breakup products, and may be unexpectedly large. The positronium (Ps) formation channel being open at the direct ionization threshold may also complicate considerations. Overall, theoretically, there has been little agreement on the range of validity of the Wannier approach when applied to positrons, with classical-trajectory Monte Carlo (CTMC) calculations suggesting the threshold law holds up to $9 \mathrm{eV}$ excess energy [14], whereas other work suggests a range of validity of $\epsilon<0.1 \mathrm{eV}[9,10,12]$, where $\epsilon$ is excess energy above the ionization threshold.

The apparatus and experimental techniques used in the present measurements have been discussed in detail previously in Refs. [20,21], and as such will only be discussed briefly here. Positrons are obtained from a $45 \mathrm{mCi}{ }^{22} \mathrm{Na}$ source moderated with solid neon frozen at $\approx 8 \mathrm{~K}$ to a goldcoated cone surrounding the source. Moderated positrons are emitted with an energy spread of $1-2 \mathrm{eV}$, and separated from unmoderated positrons through a "bend" in the guiding magnetic field. The low-energy positrons are magnetically confined along the length of the beam line with a field of $530 \mathrm{G}$ and electrostatically guided to a Surko buffer gas trap. Here, a pulsed beam is created with an energy spread of less than $100 \mathrm{meV}$ for the present measurements. For this experiment, the trap was operated at $\approx 20 \mathrm{~Hz}$ with a temporal pulse width less than $1 \mu \mathrm{s}$. The positron beam is guided to a scattering cell containing the target gas, and the bias of this cell can be varied to set a range of scattering energies. For these measurements, the scattering cell was biased negatively with respect to the ground, so that electrons from direct ionization are ejected from the cell, as shown in Fig. 1. Downstream of the scattering cell are a retarding potential analyser (RPA) and two microchannel plates (MCPs) in a chevron configuration to detect positrons or electrons passing through the RPA, depending on the potential arrangement on the MCPs.

Possible channels for the ionization of a target atom with an incident positron beam are

$$
e^{+}+X \rightarrow \operatorname{Ps}+X^{+}
$$

and

$$
e^{+}+X \rightarrow e^{+}+e^{-}+X^{+} .
$$

The Wannier relation describes the nature of direct ionization, given by Eq. (3). The absolute, direct ionization cross section can be determined by the following equation:

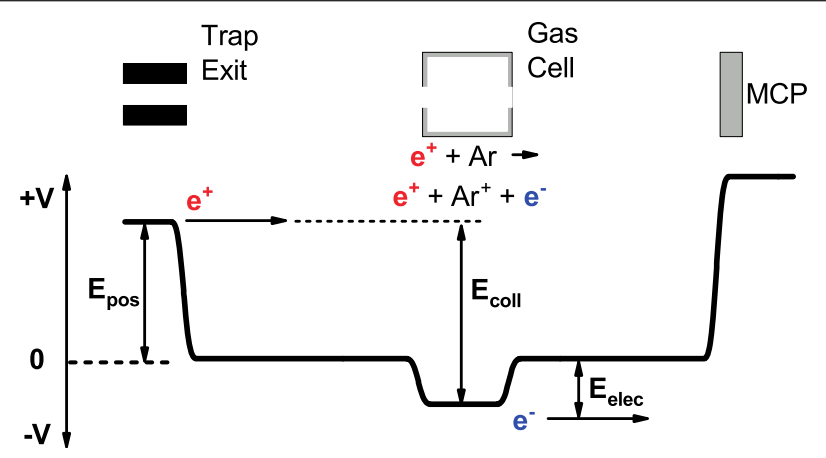

FIG. 1. Schematic of the experimental setup: $E_{\mathrm{pos}}$ is the energy of the positron beam from the trap, $E_{\text {coll }}$ is the collision energy, and $E_{\text {elec }}$ is the energy of the electron resulting from the ionization event after acceleration out of the scattering cell. Electrons are detected by the MCPs, and positrons passing through the cell are rejected and pass through the cell once more before being lost at the trap stage.

$$
\sigma_{i}=\frac{1}{n l} \frac{\epsilon_{e} I_{e}}{\epsilon_{p} I_{p}}
$$

where $I_{p}$ is the incident positron intensity and $I_{e}$ is the electron yield produced from direct ionization, $\epsilon_{e}$ is the electron detection efficiency, $\epsilon_{p}$ is the positron detection efficiency, $n$ is the number density of the target atom, and $l$ is the length of the scattering cell. However, as the positron and electron detection efficiencies are unknown in this case, the measured cross section is unnormalized. Despite this, Wannier's law is a proportionality, so a relative cross section measurement provides a sufficient test for the exponential threshold behavior of the ionization cross section. This relative cross section is now given by

$$
\sigma_{i}=\frac{1}{n l} \frac{\epsilon_{r} I_{e}}{I_{p}},
$$

where $\epsilon_{r}$ is the ratio of the detection efficiencies and is an unknown in the current measurements.

To determine the electron yield per positron in this work, a number of separate measurements are required [21]. Electron and positron intensities cannot be measured simultaneously, and the separate measurements are obtained via altering the bias to the microchannel plate. As the solid neon moderator undergoes decay, which results in a corresponding positron intensity decay, the incident positron intensity must be measured before and after the electron scan to account for any change in intensity over the course of the measurement. The decay is assumed to be linear, and therefore intensities from the start and finish of the scan are averaged. Due to the nature of the experimental arrangement, once positrons have entered the cell, undergone some scattering event, and then exited the cell, they will encounter the positively biased MCPs and be turned around. This will result in the positron beam passing through the cell a second time, and this is accounted for by taking a measurement of the positron 
intensity with the gas present in the cell. The beam is axially confined with a strong magnetic field of $530 \mathrm{G}$, which separates the electron yield into the forward $\left(0^{\circ}-90^{\circ}\right)$ and backward $\left(90^{\circ}-180^{\circ}\right)$ directions. For the first positron beam pass through the scattering cell, the forward-scattered electrons are ejected from the cell and accelerated towards the MCPs, whereas the backwards-scattered electrons are ejected from the cell in the backwards direction and lost at the trap. As stated before, the positron beam is then reflected due to the high positive potential at the MCPs, and the positron beam passes through the cell a second time. During this second pass, backward-scattered electrons with respect to the positron beam direction are accelerated towards the MCPs, whereas the forward-scattered electrons, along with the positron beam, continue and are lost at the trap. After the positron pulse first passes through the cell, the intensity is reduced due to positronium formation, which acts as a loss process in this configuration. Thus, the intensity entering the scattering cell during the second pass is reduced, and must be accounted for. This gives the equation

$$
\sigma_{i}=\frac{\epsilon_{r}}{n l} \frac{I_{e}^{f}+I_{e}^{b}}{I_{p}^{f}+I_{p}^{b}}
$$

where the superscripts $f$ and $b$ indicate the motion of the positrons forward and backward through the scattering cell, respectively. Through use of the pulsed beam, a correlated electron peak can be detected. Electron signals from the forward and backward positron pulses through the cell are shown in Fig. 2.

For the results presented here, typical pressures in the cell were 3.5 mTorr, corresponding to a scattering probability of $\simeq 30 \%$ inside the scattering cell for argon. Error values shown in the plot are the statistical uncertainty of the

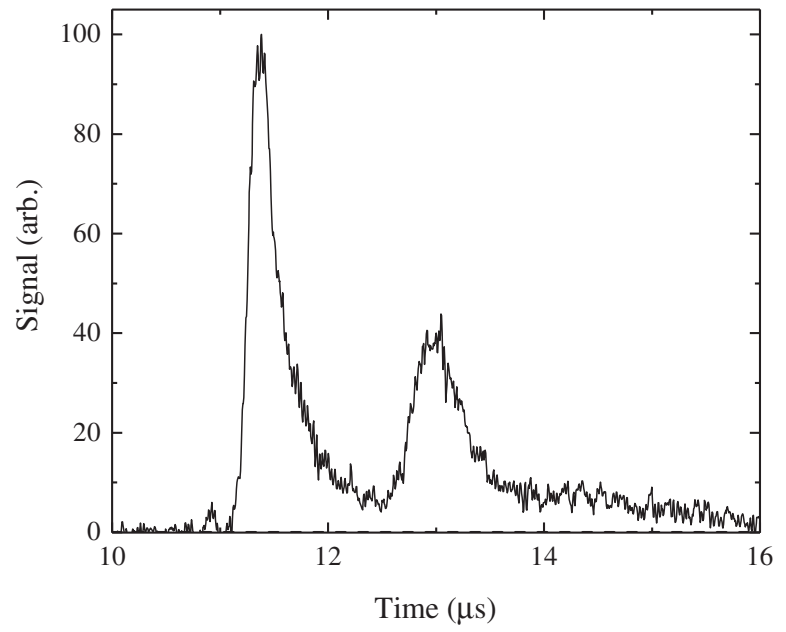

FIG. 2. The electron signal at the MCPs for a positron incident energy of $25 \mathrm{eV}$. The two peaks can be attributed to electrons in the forward (0-90 degrees) and backward (90-180 degrees) directions respectively, figure adapted from [21]. measurement. The measurement is repeated for target gas absent from the cell, to ensure no background electron signal is present.

The formation of positronium (Ps) is an ionization process in which the ionized electron is captured by the positron to become bound with the binding energy of half a Rydberg $(6.8 \mathrm{eV})$. In this energy range, ionization can proceed through a direct process [Eq. (3)] or from positronium formation [Eq. (2)]. Measuring electron yield isolates the measurement from detecting ions produced from the Ps formation. However, if the target density is sufficiently high, the Ps atom formed in the process described in Eq. (2) can undergo fragmentation via a secondary collision, also producing an electron that may be detected. The importance of multiple scattering events such as this can be assessed by checking the linearity of the cross section (electron yield) as a function of target density. For the target densities used in this experiment, the effect on the determination of the direct ionization cross section was found to be negligible.

The relative cross section for direct positron impact ionization of argon was measured, with excess energies in the range of $0<\epsilon<2 \mathrm{eV}$. These data are presented in Fig. 3. The electron ionization yield is plotted against excess energy, $\epsilon$, on a logarithmic scale, and a fit to the data performed for excess energies $<2 \mathrm{eV}$, where the lowest experimentally accessible excess energy was $220 \mathrm{meV}$. This fit gives a value of $n=1.05 \pm 0.14$ for the Wannier exponent, as given by Eq. (1). The energy range of measurements was chosen to coincide with the previously determined range of validity from electron scattering experiments, which indicate that the Wannier conditions hold for up to $2 \mathrm{eV}$ above the ionization threshold [3]. The exponent obtained in this case is different from the value of 2.65 predicted by Klar [7], and is in fact different from all

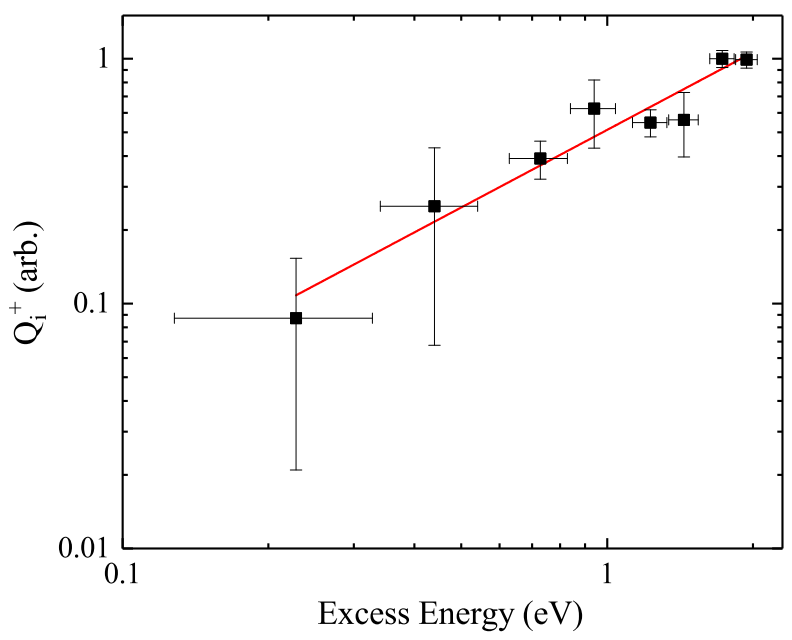

FIG. 3. Plot of threshold ionization by positron impact as a function of excess energy, for an argon target. The line of best fit is shown, and gives an exponent of $1.05 \pm 0.14$. 
other previous theoretical results. In fact, it is much closer to the exponent predicted for electron impact ionization near threshold, which gives an exponent of $1.127[1,5,6]$. It also disagrees with the exponents found in the previous experimental work by Ashley et al. [15]. Although these were for different target species, the dependance of the Wannier law on only the charge of the residual ion means that this is a valid comparison.

The reasons for this disagreement with theory are not clear. Argon is a more complex target than has been typically investigated for studies of the Wannier threshold law, with the spin-orbit interaction leading to an ion core energy splitting of $177 \mathrm{meV}$ [22], and thus two ionization thresholds. This, in the semiclassical treatment, would lead to a summing of the power laws as follows:

$$
Q_{i}^{-} \propto A\left(E_{m}-E_{i}\right)^{n}+B\left[E_{m}-\left(E_{i}+177 \mathrm{meV}\right)\right]^{n},
$$

where $A$ and $B$ are constants. Fitting was undertaken using both Eqs. (1) and (7), and there was no significant difference observed for the exponent in both cases.

It may be that the range of validity for the Wannier conditions is much lower than expected, requiring detailed measurements much closer to threshold to determine the appropriate exponent. Restricting the data measured here to only those points up to $1 \mathrm{eV}$ above threshold results in an exponent of $n=1.40 \pm 0.20$, which is only slightly different from the value for the full range of data, and still far lower than the value of 2.65 predicted. This may suggest a significantly reduced range of validity, if this is in fact the explanation for the discrepancy. In this case, with the current experimental apparatus and procedure, it would be very difficult to further probe the near-threshold region with the accuracy and precision required to test a greatly reduced threshold region.

The comparison with the electron threshold law is provocative, with the result found in this measurement consistent with that found both experimentally and theoretically for the electron value of $n=1.127$ for double escape from a singly ionized core. This would support Temkin's assertion that the relation for ionization with positron and electrons will have the same form [9]; however, with the limited energy resolution of these results, and given the present statistical variations, we cannot reliably fit to the modulated linear law. It may be that revisiting this problem with further calculations, and more experimental measurements, resolve some of the discrepancies.

We have presented the first detailed experimental measurements of near threshold positron impact ionization with significantly improved energy resolution from what has previously been available. When fitted with a power law, a slope of $1.05 \pm 0.14$ was obtained. This disagrees with theoretical predictions of Klar and others, who predict the ionization cross section to vary as in Eq. (1), with an exponent of $n=2.65$. This result is consistent with some previous measurements [16,17]; however, it is in disagreement with the measurements of Ashley et al. [15]. We note that none of these previous measurements have had a significant component of their data in the conventionally accepted near-threshold region. The reasons for the disagreement with theoretical predictions is unclear, but this may suggest that the Wannier regime is constrained much more closely to threshold than previously anticipated, or in fact that the threshold escape law is the same for both electron and positron impact.

The authors would like to thank Professors Tim Gay and Ravi Rau for valuable discussions regarding the research presented here. We would also like to thank the input of the referees for valuable suggestions incorporated into the final version of this paper. This work was supported by the Australian Research Council's Discovery and Discovery Early Career Researcher Programs. We would also like to thank Ross Tranter, Colin Dedman, and Luke Materne for their expertise and technical support.

*Corresponding author. james.sullivan@anu.edu.au

[1] G. H. Wannier, Phys. Rev. 90, 817 (1953).

[2] S. Cvejanovic and F. Read, J. Phys. B 7, 1841 (1974).

[3] H. Kossmann, V. Schmidt, and T. Andersen, Phys. Rev. Lett. 60, 1266 (1988).

[4] R. I. Hall, A. G. McConkey, L. Avaldi, K. Ellis, M. A. MacDonald, G. Dawber, and G. C. King, J. Phys. B 25, 1195 (1992).

[5] R. Peterkop, J. Phys. B 4, 513 (1971).

[6] A. R. P. Rau, Phys. Rev. A 4, 207 (1971).

[7] H. Klar, J. Phys. B 14, 4165 (1981).

[8] P. Grujic, J. Phys. B 15, 1913 (1982).

[9] A. Temkin, J. Phys. B 15, L301 (1982).

[10] A. Temkin, Phys. Rev. Lett. 49, 365 (1982).

[11] J. M. Rost and E. J. Heller, Phys. Rev. A 49, R4289 (1994).

[12] A. Temkin, Phys. Rev. A 30, 2737 (1984).

[13] S. Geltman, J. Phys. B 16, L525 (1983).

[14] A. E. Wetmore and R. E. Olson, Phys. Rev. A 34, 2822 (1986).

[15] P. Ashley, J. Moxom, and G. Laricchia, Phys. Rev. Lett. 77, 1250 (1996).

[16] H. Knudsen, L. Brun-Nielsen, M. Charlton, and M. Poulsen, J. Phys. B 23, 3955 (1990).

[17] O. Sueoka, B. Jin, and A. Hamada, Appl. Surf. Sci. 85, 59 (1995).

[18] H. Klar, J. Phys. B 14, 3255 (1981).

[19] J. M. Feagin, J. Phys. B 17, 2433 (1984).

[20] J. Sullivan, A. Jones, P. Caradonna, C. Makochekanwa, and S. Buckman, Rev. Sci. Instrum. 79, 113105 (2008).

[21] J. R. Machacek, T. J. Babij, D. J. Murtagh, S. J. Buckman, and J. P. Sullivan, Eur. Phys. J. D 71, 328 (2017).

[22] A. Kramida, Yu. Ralchenko, and J. Reader (NIST ASD Team), NIST Atomic Spectra Database (ver. 5.3), (National Institute of Standards and Technology, Gaithersburg, MD, 2015), http://physics.nist.gov/asd. 